\title{
Resveratrol Ameliorates Experimental Autoimmune Myocarditis
}

\author{
Yuki Yoshida, MD; Tetsuo Shioi, MD; Tohru Izumi, MD
}

\begin{abstract}
Background Myosin-induced autoimmune myocarditis of rats is a model of human dilated cardiomyopathy. Resveratrol is a natural polyphenol found in grapes and wine that is reported to have cardioprotective and immunomodulatory effects.

Methods and Results To examine the effect of resveratrol on myocarditis, vehicle or resveratrol $(50 \mathrm{mg} / \mathrm{kg}$ per day) was administered to cardiac myosin immunized rats 1 day before the immunization. At 14 days after immunization, resveratrol had preserved cardiac function of myosin-immunized rats according to echocardiographic analysis. The heart weight/tibial length ratio of vehicle-treated myosin-immunized rats was increased by 1.8-fold compared with unimmunized rats, and resveratrol attenuated the heart weight increase. Resveratrol significantly decreased cellular infiltration, fibrosis, and expression of inflammatory cytokines in the myocardium. Expressions of antioxidant genes were increased in myosin-immunized hearts, and resveratrol decreased those expressions. Resveratrol also attenuated myocarditis 21 days after immunization. SIRT1, a potential effector of resveratrol, was increased in the myocardium of myosin-immunized rats compared with unimmunized rats. The SIRT1 protein was localized mainly in infiltrating mononuclear cells.

Conclusions Resveratrol significantly ameliorated myocardial injury and preserved cardiac function in a rat model of autoimmune myocarditis. Resveratrol may be a therapeutic modality for myocarditis. (Circ J 2007; 71: $397-404)$
\end{abstract}

Key Words: Aging; Heart failure; Myocarditis; Resveratrol

$\mathbf{P}$ atients with myocarditis present a variety of clinical manifestations, such as heart failure, arrhythmia, or circulatory collapse!,2 Although myocarditis often follows viral infection, its pathogenesis is not fully understood. There is substantial evidence suggesting that autoimmune responses to heart antigens, particularly cardiac myosin, following viral infection may contribute to the disease process4 An experimental model of autoimmune myocarditis (EAM) in Lewis rats produced by immunization with cardiac myosin is characterized by extremely severe myocardial lesions and multinucleated giant cells, 5,6 and it has been reported that the pathogenesis of both human giant-cell myocarditis and viral myocarditis resembles that of EAM?

Resveratrol is an active ingredient of Polygonum capsidatum, a plant known for its medical use, as well as being present in peanuts and grapevines. The presence of resveratrol in red wine raises interest in this compound, because the consumption of red wine is known to reduce the risk of cardiovascular diseases? The biological effects of resveratrol include protection of cells from lipid accumulation, chemoprevention, immunomodulation, antiproliferation, and differentiation, 10 Recent focus has been on the use of resveratrol in the treatment of cardiovascular disease because it has potent cardioprotective properties against ischemia-reperfusion injury in rat hearts, ${ }^{11,12}$ as well as

(Received August 17, 2006; revised manuscript received November 28, 2006; accepted December 5, 2006)

Department of Internal Medicine and Cardiology, Kitasato University School of Medicine, Sagamihara, Japan

Mailing address: Tetsuo Shioi, MD, Department of Cardiovascular Medicine, Graduate School of Medicine, Kyoto University, 54 Kawahara-cho, Shogoin, Sakyo-ku, Kyoto 606-8507, Japan. E-mail: tshioi@kuhp.kyoto-u.ac.jp inhibiting angiotensin II-increased cardiomyocyte hypertrophy ${ }^{13}$ and enhancing myocardial angiogenesis, both in vivo and in vitro, by induction of vascular endothelial growth factor ${ }^{14}$

Silent information regulator 2 (Sir2) is a member of the sirtuin deacetylase family of enzymes that removes acetyl groups from lysine residues in histones and other proteins in a NAD-dependent manner!5,16 SIRT1 is a mammalian ortholog of the Sir2 protein. Caloric restriction extends the life span of animals and a recent study has identified Sir2 as mediating the effects of caloric restriction ${ }^{17}$ Overexpression of Sir2 extends the lifespan in model organisms. SIRT1 deacetylates a variety of proteins, inducing $\mathrm{p} 53$, and has cytoprotective effects $!^{8}$ It has been reported that resveratrol may modulate $\operatorname{Sir} 2$ activity 19

Because resveratrol has both cardioprotective and immunomodulatory effects, it may favorably modulate the myocardial injury induced by myocarditis, which we investigated in a rat model of EAM.

\section{Methods}

Animals

Female Lewis rats (8 weeks old) were purchased from Charles River (Kanagawa, Japan). Animal care and experimental protocol were approved by the Institutional Animal Care and Use Committee of Kitasato University.

\section{Immunization}

Antigen was prepared from porcine hearts by previously described procedures ${ }^{20}$ The cardiac myosin was emulsified with an equal volume of complete Freund's adjuvant supplemented with Mycobacterium tuberculosis H37Ra (Difco, Detroit, MI, USA) to a final concentration of $5 \mathrm{mg} / \mathrm{ml}$. The 
emulsified solution $(0.25 \mathrm{ml})$ or adjuvant alone was subcutaneously injected into the footpads of rats. The day of myosin injection was defined as day 0 .

\section{Experimental Protocols}

Resveratrol (Sigma, St Louis, MO, USA: lot number 065K5203) was dissolved in dimethylsulfoxide (DMSO) at concentration of $20 \mathrm{mg} / \mathrm{ml}$, and $0.5 \mathrm{ml}$ of resveratrol dissolved in DMSO or the same amount of DMSO alone was injected intraperitoneally into the rats once daily. The dose of resveratrol was equivalent to $50 \mathrm{mg} / \mathrm{kg}$.

Protocol 1 Vehicle or resveratrol was administered to rats from 1 day prior to immunization to 14 days afterward. The rats were killed after echocardiographic examination 14 days after immunization. Body weight, heart weight, and tibial length were measured. The hearts were used for analysis of histology, gene expression, and protein expression.

Protocol 2 Vehicle or resveratrol was started 1 day before immunization and continued until echocardiographic examination 21 days later, after which the rats were killed and the same measurements taken.

Protocol 3 Vehicle or resveratrol was started 1 day after immunization, continued for 14 days and then the rats were killeded after echocardiographic examination. The weights and tibial length were measured as per the other 2 protocols.

\section{Heart Rate (HR) and Blood Pressure (BP)}

Systolic BP (SBP) and HR were recorded by the tail cuff method (Softron BP-98A, Tokyo, Japan) ${ }^{21}$ For measuring $\mathrm{SBP}$, rats were placed in a warm chamber maintained at $40^{\circ} \mathrm{C}$ for $10 \mathrm{~min}$ in an individual Plexiglas restrainer. Three pressure measurements were recorded for each rat, and the average SBP was calculated.

\section{Echocardiography}

Transthoracic echocardiographic analysis was performed using a ProSound SSD-4000 (ALOKA, Tokyo, Japan) with a $7.5-\mathrm{MHz}$ imaging transducer. 2-2-2 tribromoethanol (Aldrich, $0.4-0.6 \mathrm{mg} / \mathrm{kg}$ ) was used for anesthesia. M-mode echocardiography was performed at the papillary muscle level, and the left ventricular end-diastolic dimension, endsystolic dimension and left ventricular posterior wall thickness were measured; fractional shortening (FS) was calculated as described 22

\section{Western Blotting}

The hearts were removed and immediately frozen in liquid nitrogen. Lysates were obtained by homogenization of tissue in ice-cold buffer as described ${ }^{22}$ Cardiac tissue lysates were subjected to sodium dodecyl sulfate polyacrylamide gel electrophoresis, and the proteins were transferred onto polyvinylidene difluoride membranes as described 22 For analysis of SIRT1, the blots were probed with the antiSir2 antibody (1:2,000 Upstate Biotechnology, Lake Placid, NY, USA) or anti-glyceraldehye-3-phosphate dehydrogene antibody (GAPDH; 1:5,000, Research Diagnosis, Flandeuns, NJ, USA).

\section{Quantitative Reverse Transcription (RT)-Polymerase Chain Reaction (PCR)}

RNA was prepared from heart tissue by the acid-guanidinium-phenol chloroform method. cDNA was synthesized using the SuperScript first-strand synthesis system (Invitrogen, Carlsbad, CA, USA) and then subjected to PCR with SYBR green (Applied Biosystems, Foster City, CA, USA) as the detected fluoroprobe. Incorporation of the SYBR green dye into the PCR products was monitored in a real-time manner with the ABI PRISM 7900HT Sequence Detection System, and the threshold cycle, which defines the PCR cycle at which exponential growth of PCR products begins, was calculated. The PCR reactions were cycled 35 times by a 3 -step cycle procedure (denaturation $95^{\circ} \mathrm{C}, 15 \mathrm{~s}$; annealing $60^{\circ} \mathrm{C}$, $30 \mathrm{~s}$; extension $72^{\circ} \mathrm{C}, 30 \mathrm{~s}$ ). mRNA levels of B-type natriuretic peptide (BNP), tumor necrosis factora (TNFa), inducible nitric oxide synthase (iNOS), manganese superoxide dismutase (Mn-SOD), copper/zinc superoxide dismutase (Cu/Zn-SOD), and SIRT1 were measured. Each of the RNA samples was normalized with an endogenous control (GAPDH mRNA). The primers used for the PCR analysis was designed using OLIGO Primer Analysis Software (TaKaRa, Kyoto, Japan) and oligonucleotides were synthesized at Invitrogen (Tokyo, Japan). The primer sequences were as follows:

BNP; sense 5'-TTCCGGATCCAGGAGAGACTT-3', antisense 5'-CCTAAAACAACCTCAGCCCGT-3', SIRT1; sense 5'-TTGGCACCGATCCTCGAA-3', antisense 5'ACAGAAACCCCAGCTCCA-3',TNFa; sense 5'-TGATCGGTCCCAACAAGGA-3', antisense 5'-TGCTTGGTGGTTTGCTACGA-3', iNOS; sense 5'-GAAAACCCCAGGTGCTATTCC-3', antisense 5'-CATTCTGTGCAGTCCCAGTGA-3', Mn-SOD sense 5'-ACCACGCGACCTACGTGAAC-3', antisense 5'-TGCAGGCTGAAGAGCAACCT-3', Cu/Zn-SOD sense 5'-CGAGCATGGGTTCCATGTC-3', antisense 5'-CTGGACCGCCATGTTTCTTAG3',GAPDH sense 5'-AGGTCGGTGTGAACGGATTTG-3', antisense 5'-TGACTGTGCCGTTGAACTTGC-3'.

\section{Histopathology}

The rats were killed on day 14 and their hearts were fixed in $3.7 \%$ formaldehyde, embedded in paraffin and sectioned. The sections were stained by the hematoxylin-eosin or Mallory-Azan method and the microscopic findings were expressed as infiltration and fibrosis scores for the respective stains. Morphometric quantification was performed with Mac-Scope Image software (version 2.59; Mitani Corp, Fukui, Japan) on a Macintosh computer 23 The outline of the area of cell infiltration or fibrosis was traced by hand, the profile area was assessed automatically and the area ratio (affected area/total area in percent) was calculated.

\section{Immunohistochemical Analysis of SIRT1 Expression}

Heart tissue was embedded in OCT compound, snapfrozen on dry ice, and stored at $-70^{\circ} \mathrm{C}$. Samples were sectioned on a cryostat at $10 \mu \mathrm{m}$ and then fixed for $5 \mathrm{~min}$ in acetone at $4^{\circ} \mathrm{C}$. The sections were incubated with anti-SIRT1 antibody (rabbit IgG, Santa Cruz Biotechnology, Santa Cruz, CA, USA) and anti-actinin antibody (mouse IgG, Sigma) at $4{ }^{\circ} \mathrm{C}$ overnight. Next, sections were incubated with fluorescent isothiocyanate-labeled anti-rabbit IgG and rhodaminelabeled anti-mouse IgG antibodies at room temperature for $30 \mathrm{~min}$. Nuclei were stained using TOTO-3 (Invitorogen Carlsbad, CA, USA). Sections were analyzed by confocal microscopy (Carl Zeiss, Oberkochen, Germany).

\section{Statistical Analysis}

All values are expressed as mean \pm SEM. Differences between groups were compared using Student's t-tests. A p-value $<0.05$ was considered as significant. 
Table 1 Blood Pressure and HR of EAM Rats Treated With Resveratrol for 2 Weeks

\begin{tabular}{lcccccc}
\hline \hline & \multicolumn{2}{c}{ Unimmunized } & & \multicolumn{2}{c}{ Myosin-immunized } \\
\cline { 2 - 3 } \cline { 6 - 7 } & Vehicle & Resveratrol & & \multicolumn{2}{c}{ Vehicle } & Resveratrol \\
\hline No. animals & 5 & 5 & & 8 & 8 \\
SBP $(\mathrm{mmHg})$ & $115.2 \pm 3.9$ & $118.5 \pm 6.6$ & & $96.4 \pm 5.1^{*}$ & $108.8 \pm 6.4$ \\
HR (beats $/$ min $)$ & $323.7 \pm 10.7$ & $353.9 \pm 16.2$ & & $372.3 \pm 14.1^{*}$ & $397.2 \pm 14.7$ \\
\hline
\end{tabular}

Resveratrol was started 1 day before immunization, and given daily for 2 weeks. Results are mean \pm SEM.

${ }^{*} p<0.05$ vs unimmunized rats treated with vehicle or resveratrol.

$H R$, heart rate; EAM, experimental model of autoimmune myocarditis; SBP, systolic blood pressure.

Table 2 Echocardiographic Data of EAM Rats Treated With Resveratrol for 2 Weeks

\begin{tabular}{|c|c|c|c|c|}
\hline & \multicolumn{2}{|c|}{ Unimmunized } & \multicolumn{2}{|c|}{ Myosin-immunized } \\
\hline & Vehicle & Resveratrol & Vehicle & Resveratrol \\
\hline No. animals & 5 & 5 & 8 & 6 \\
\hline$H R($ beats/min $)$ & $444 \pm 13$ & $429 \pm 6$ & $405 \pm 9 *$ & $385 \pm 10 *$ \\
\hline Diastolic posterior wall thickness ( $\mathrm{mm}$ ) & $1.54 \pm 0.12$ & $1.62 \pm 0.16$ & $1.85 \pm 0.13$ & $1.64 \pm 0.08$ \\
\hline LV diastolic diameter $(\mathrm{mm})$ & $4.70 \pm 0.11$ & $4.78 \pm 0.17$ & $4.56 \pm 0.15$ & $4.51 \pm 0.24$ \\
\hline LV systolic diameter $(\mathrm{mm})$ & $1.46 \pm 0.11$ & $1.36 \pm 0.11$ & $2.51 \pm 0.17 *$ & $1.91 \pm 0.22 \dagger$ \\
\hline$F S(\%)$ & $68.5 \pm 3.0$ & $71.6 \pm 1.7$ & $44.6 \pm 3.4 *$ & $58.2 \pm 3.0 *, \dagger$ \\
\hline
\end{tabular}

Resveratrol was started 1 day before immunization, and given daily for 2 weeks.

$L V$, left ventricle; FS, fractional shortening. Other abbreviations see in Table 1.

Results are mean \pm SEM.

${ }^{*} p<0.05$ vs unimmunized rats treated with vehicle or resveratrol, ${ }^{\dagger} p<0.05$ vs vehicle-treated myosin-immunized rats.

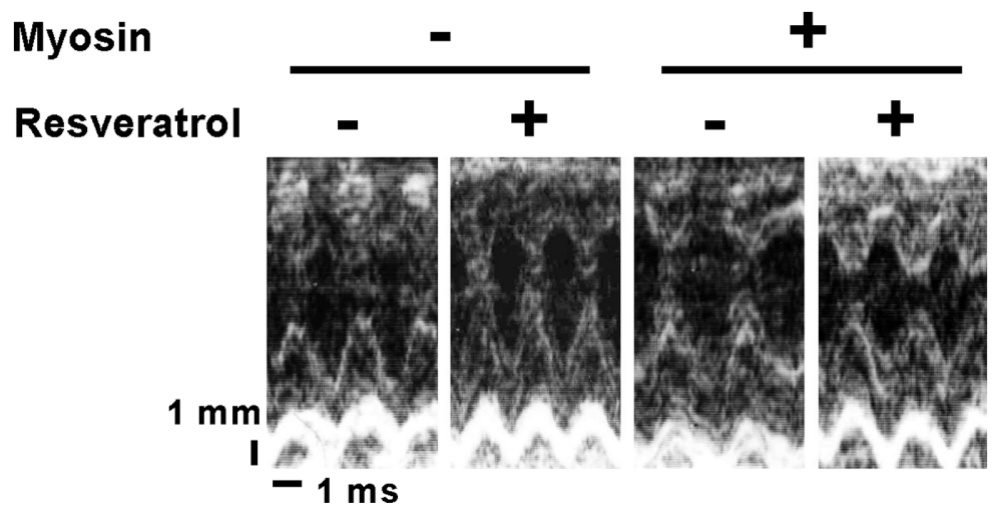

Fig 1. Echocardiographic analysis of experimental model of autoimmune myocarditis rats treated with resveratrol. (Left to right) Vehicle-treated unimmunized rat, resveratrol-treated unimmunized rat, vehicle-treated myosin-immunized rat, resveratrol-treated myosinimmunized rat. Fractional shortening of vehicle-treated myosin-immunized rats was decreased compared with vehicle-treated unimmunized rats. Resveratrol treatment attenuated the decrease of fractional shortening in the myosin-immunized rats.

Table 3 Postmortem Analysis of EAM Rats Treated With Resveratrol for 2 Weeks

\begin{tabular}{|c|c|c|c|c|}
\hline & \multicolumn{2}{|c|}{ Unimmunized } & \multicolumn{2}{|c|}{ Myosin-immunized } \\
\hline & Vehicle & Resveratrol & Vehicle & Resveratrol \\
\hline No. animals & 5 & 5 & 8 & 8 \\
\hline Body weight $(g)$ & $177.2 \pm 2.0$ & $182.2 \pm 4.3$ & $171.0 \pm 3.1$ & $184.4 \pm 2.3^{\dagger}$ \\
\hline Heart weight (mg) & $590.4 \pm 6.1$ & $589.6 \pm 16.0$ & $1,023.6 \pm 86.4 *$ & $739.4 \pm 60.9^{\dagger}$ \\
\hline Lung weight (mg) & $1,045.2 \pm 31.1$ & $1,004.6 \pm 55.9$ & $1,039.3 \pm 26.7$ & $1,013.0 \pm 14.3$ \\
\hline Tibial length $(\mathrm{mm})$ & $33.6 \pm 0.2$ & $33.2 \pm 0.1$ & $32.1 \pm 0.2 *$ & $32.3 \pm 0.2 *$ \\
\hline Heart weight/body weight $(\mathrm{mg} / \mathrm{g})$ & $3.34 \pm 0.07$ & $3.23 \pm 0.04$ & $6.05 \pm 0.58 *$ & $4.04 \pm 0.99 \dagger$ \\
\hline Lung weight/body weight (mg/g) & $5.90 \pm 0.18$ & $5.51 \pm 0.25$ & $6.08 \pm 0.14$ & $5.50 \pm 0.07$ \\
\hline Heart weight/tibial length $(\mathrm{mg} / \mathrm{mm})$ & $17.6 \pm 0.2$ & $17.8 \pm 0.4$ & $31.9 \pm 2.6^{*}$ & $22.9 \pm 1.9 *, \dagger$ \\
\hline Lung weight/tibial length $(\mathrm{mg} / \mathrm{mm})$ & $31.1 \pm 0.8$ & $30.3 \pm 1.8$ & $32.4 \pm 0.7$ & $31.4 \pm 0.4$ \\
\hline
\end{tabular}

Resveratrol was started 1 day before immunization, and given daily for 2 weeks. Abbreviation see in Table 1. Results are mean \pm SEM.

${ }^{*} p<0.05$ vs unimmunized rats treated with vehicle or resveratrol, ${ }^{t} p<0.05$ vs vehicle-treated myosin-immunized rats.

\section{Results}

\section{$H R$ and $B P$}

At 14 days after immunization, we examined the effect of resveratrol on HR and BP. The SBP of vehicle-treated myosin-immunized rats was decreased compared with that of vehicle-treated unimmunized rats $(96.4 \pm 5.1 \mathrm{mmHg}$ vs $115.2 \pm 3.9 \mathrm{mmHg}, \mathrm{p}<0.05$, Table 1). The BP of resveratroltreated myosin-immunized rats was not different from that of vehicle-treated unimmunized rats. 


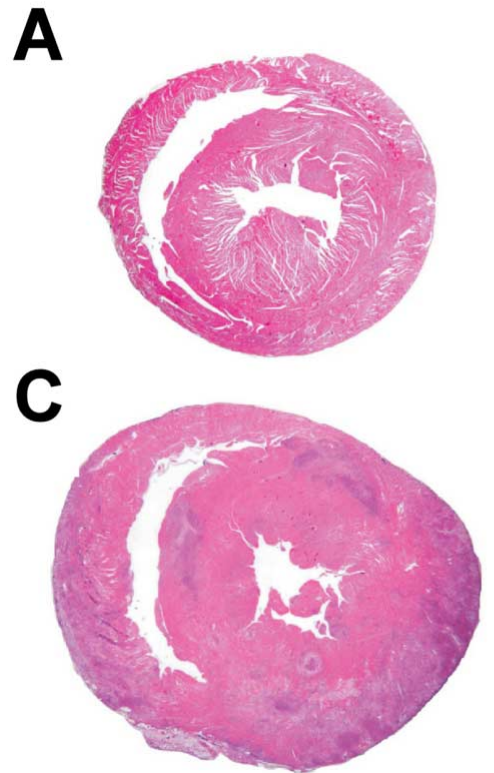

B

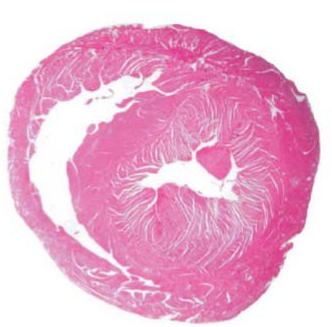

D

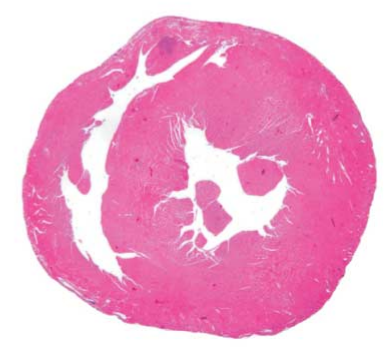

Fig 2. Macroscopic analysis of experimental model of autoimmune myocarditis rats treated with resveratrol. (A) Vehicle-treated unimmunized rat, (B) resveratrol-treated unimmunized rat, $(\mathrm{C})$ vehicletreated myosin-immunized rat, and (D) resveratroltreated myosin-immunized rat. The left ventricle was dilated and wall thickness was increased in vehicletreated myosin-immunized rats. Resveratrol attenuated cardiac enlargement. $\mathrm{Bar}=1 \mathrm{~mm}$.
A
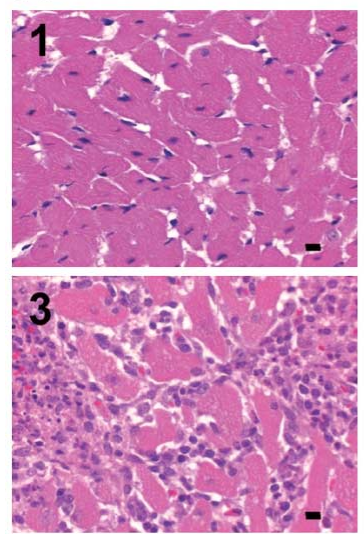

B
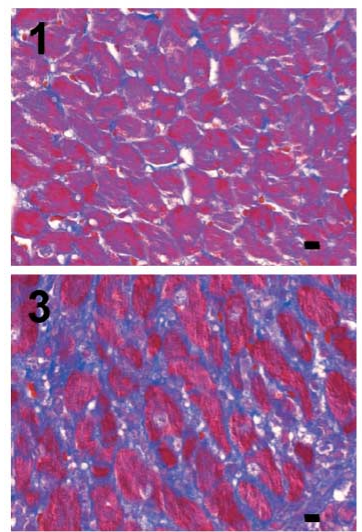
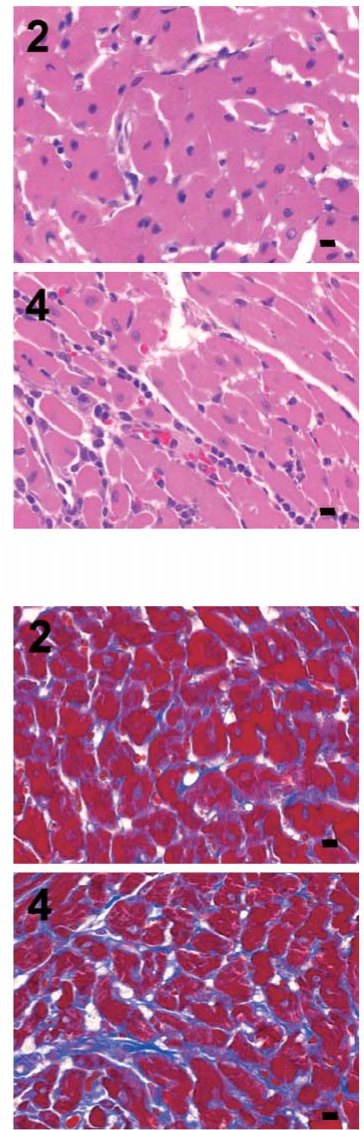

Fig 3. Effect of resveratrol on (A) cellular infiltration and (B) fibrosis. (A) Representative images of (1) vehicle-treated unimmunized rats, (2) resveratrol-treated unimmunized rats, (3) vehicle-treated myosin-immunized rats, and (4) resveratrol-treated myosin-immunized rats. Resveratrol attenuated cellular infiltration. Bars $=10 \mu \mathrm{m}$. (B) Representative images of (1) vehicle-treated unimmunized rats, (2) resveratrol-treated unimmunized rats, (3) vehicle-treated myosinimmunized rats, and (4) resveratrol-treated myosin-immunized rats, are shown. Resveratrol attenuated fibrosis. Bars $=10 \mu \mathrm{m}$.

\section{Echocardiographic Analysis}

At 14 days after immunization, we examined the effect of resveratrol on cardiac function using echocardiography (Table2, Fig 1). The FS of vehicle-treated myosin-immunized rats was decreased compared with that of vehicletreated unimmunized rats $(44.6 \pm 3.4 \%$ vs $68.5 \pm 3.0 \%, \mathrm{p}<$ $0.05)$. Resveratrol treatment attenuated the decrease in FS in myosin-immunized rats, thus preserving the cardiac function of EAM rats.

\section{Postmortem Analysis}

At 14 days after immunization, the heart weight/tibial length ratio of myosin-immunized rats was increased by 1.81-fold compared with vehicle-treated unimmunized rats $(31.9 \pm 2.6 \mathrm{mg} / \mathrm{mm}$ vs $17.6 \pm 0.2 \mathrm{mg} / \mathrm{mm}, \mathrm{p}<0.05$, Table 3 , Fig 2). In contrast, the heart weight/tibial length ratio of resveratrol-treated myosin-immunized rats was increased by 1.29 -fold compared with resveratrol-treated unimmunized rats $(22.9 \pm 1.9 \mathrm{mg} / \mathrm{mm}$ vs $17.8 \pm 0.4 \mathrm{mg} / \mathrm{mm}, \mathrm{p}<0.05)$. Thus, resveratrol significantly suppressed the increase in heart weight of EAM rats.

\section{Histopathological Analysis}

The area of cell infiltration in resveratrol-treated myosinimmunized rats was significantly decreased compared with vehicle-treated myosin-immunized rats $(2.6 \pm 1.5 \%$ vs $26.4 \pm$ $3.6 \%$, p $<0.05$; Fig 3, Table 4). The area of fibrosis in resveratrol-treated myosin-immunized rats was decreased from that of vehicle-treated myosin-immunized rats $(5.5 \pm 3.2 \%$ vs $28.0 \pm 4.6 \%$, p<0.05; Fig 3, Table 4). Overall, resveratrol attenuated the inflammation and fibrosis of the myocardium of EAM rats.

\section{BNP Gene Expression}

BNP mRNA of myosin-immunized rats was increased by $12.4 \pm 2.2$-fold compared with vehicle-treated unimmunized rats (Table 5). BNP mRNA of resveratrol-treated myosin-immunized rats was increased by $1.6 \pm 0.5$-fold compared with vehicle-treated unimmunized rats. Resveratrol significantly suppressed the increase in BNP mRNA in the myocardium of EAM rats. 
Table 4 Cellular Infiltration and Fibrosis of the Myocardium of EAM Rats Treated With Resveratrol for 2 Weeks

\begin{tabular}{lcc}
\hline \hline & \multicolumn{2}{c}{ Myosin-immunized } \\
\cline { 2 - 3 } & Vehicle & Resveratrol \\
\hline No. animals & 6 & 6 \\
Cellular infiltration area ratio (\%) & $26.4 \pm 3.6$ & $2.6 \pm 1.5^{*}$ \\
No. animals & 6 & 6 \\
Fibrosis area ratio $(\%)$ & $28.0 \pm 4.6$ & $5.5 \pm 3.2 *$ \\
\hline
\end{tabular}

Resveratrol was started 1 day before immunization, and given daily for 2 weeks.

Abbreviation see in Table 1. Results are mean \pm SEM.

${ }^{*} p<0.05$ vs vehicle-treated myosin-immunized rats.

Table 5 BNP mRNA in the Myocardium of EAM Rats Treated With Resveratrol for 2 Weeks

\begin{tabular}{lccccc}
\hline \hline & \multicolumn{2}{c}{ Unimmunized } & & \multicolumn{2}{c}{ Myosin-immunized } \\
\cline { 2 - 3 } \cline { 5 - 6 } & Vehicle & Resveratrol & & Vehicle & Resveratrol \\
\hline No. animals & 4 & 4 & 5 & 5 \\
BNP mRNA (units) & $1.00 \pm 0.25$ & $0.41 \pm 0.13$ & & $12.42 \pm 2.22^{*}$ & $1.61 \pm 0.46^{\dagger}$ \\
\hline
\end{tabular}

Resveratrol was started 1 day before immunization, and given daily for 2 weeks.

The mean value of vehicle-treated unimmunized rats was defined as one unit.

$B N P, B$-type natriuretic peptide. Other abbreviation see in Table 1. Results are mean \pm SEM.

${ }^{*} p<0.05 v$ s unimmunized rats treated with vehicle or resveratrol, ${ }^{\dagger} p<0.05 \mathrm{v}$ vehicle-treated myosin-immunized rats.

Table 6 TNFa and iNOS mRNA in the Myocardium of EAM Rats Treated With Resveratrol for 2 Weeks

\begin{tabular}{|c|c|c|c|c|}
\hline & \multicolumn{2}{|c|}{ Unimmunized } & \multicolumn{2}{|c|}{ Myosin-immunized } \\
\hline & Vehicle & Resveratrol & Vehicle & Resveratrol \\
\hline No. animals & 5 & 5 & 5 & 5 \\
\hline$T N F \boldsymbol{a}$ mRNA (units) & $1.00 \pm 0.16$ & $1.30 \pm 0.16$ & $46.18 \pm 14.4 *$ & $1.54 \pm 0.25^{\dagger}$ \\
\hline iNOS mRNA (units) & Undetectable & Undetectable & $283.82 \pm 115.81 *$ & $1.00 \pm 0.40 *, t$ \\
\hline
\end{tabular}

Resveratrol was started 1 day before immunization, and given daily for 2 weeks.

The mean value of vehicle-treated unimmunized rats was defined as 1 unit for TNF a mRNA.

The mean value of resveratrol-treated immunized rats was defined as 1 unit for iNOS mRNA.

$T N F$ a, tumor necrosis factora; iNOS, inducible nitric oxide synthase. Other abbreviation see in Table 1.

Results are mean \pm SEM.

${ }^{*} p<0.05$ v unimmunized rats treated with vehicle or resveratrol, ${ }^{\dagger} p<0.05 v$ v vehicle-treated myosin-immunized rats.

Table 7 Mn-SOD and Cu/Zn-SOD mRNA in the Myocardium of EAM Rats Treated With Resveratrol for 2 Weeks

\begin{tabular}{lcccccc}
\hline \hline & \multicolumn{2}{c}{ Unimmunized } & & \multicolumn{2}{c}{ Myosin-immunized } \\
\cline { 2 - 3 } \cline { 5 - 6 } & Vehicle & Resveratrol & & Vehicle & Resveratrol \\
\hline No. animals & 5 & 5 & & 5 & 5 \\
Mn-SOD mRNA (units) & $1.00 \pm 0.24$ & $0.94 \pm 0.33$ & & $7.51 \pm 1.39^{*}$ & $1.42 \pm 0.35^{\dagger}$ \\
Cu/Zn-SOD mRNA (units) & $1.00 \pm 0.07$ & $1.10 \pm 0.36$ & & $3.03 \pm 1.11^{*}$ & $1.47 \pm 0.43^{\dagger}$ \\
\hline
\end{tabular}

Resveratrol was started 1 day before immunization, and was given daily for 2 weeks.

The mean value of vehicle-treated unimmunized rats was defined as 1 unit.

$\mathrm{Mn}$-SOD, manganese superoxide dismutase; $\mathrm{Cu} / \mathrm{Zn}$-SOD, copper/zinc superoxide dismutase. Other abbreviation see in Table 1.

Results are mean \pm SEM.

${ }^{*} p<0.05$ vs unimmunized rats treated with vehicle or resveratrol, ${ }^{\dagger} p<0.05 v$ v vehicle-treated myosin-immunized rats.

Expression of Inflammatory Markers

The levels of both TNFa and iNOS mRNA were analyzed using quantitative RT-PCR (Table 6). TNFa mRNA was increased by $46.2 \pm 14.4$-fold, and resveratrol suppressed TNFa expression. iNOS mRNA was increased in EAM rats and resveratrol significantly decreased its expression.

\section{Expression of Antioxidant Genes}

The level of expression of the antioxidant enzymes, Mn$\mathrm{SOD}$ and $\mathrm{Cu} / \mathrm{Zn}-\mathrm{SOD}$, was analyzed using quantitative RT-PCR (Table 7). Mn-SOD mRNA increased by 7.5 $\pm 1.4-$ fold, and $\mathrm{Cu} / \mathrm{Zn}-\mathrm{SOD}$ mRNA by 3.0 \pm 1.1 -fold in the myocardium of EAM rats. Resveratrol significantly attenuated the expression of both enzymes.

\section{SIRT1 Expression}

The level of SIRT 1 mRNA was analyzed using quantitative RT-PCR. SIRT1 mRNA was increased by $2.2 \pm 0$.2-fold in vehicle-treated myosin-immunized rats compared with vehicle-treated unimmunized rats (Fig 4A). The amount of SIRT1 protein was also increased in vehicle-treated myosin-immunized rats (Fig 4B). The immunofluorescence study showed that the SIRT1 protein was mainly localized in infiltrating mononuclear cells (Fig 5). 
A

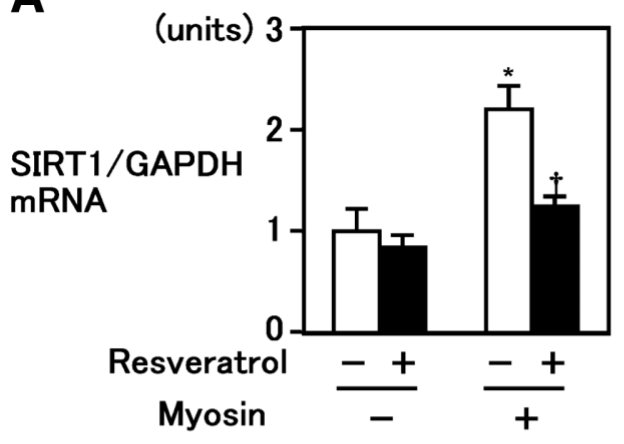

B

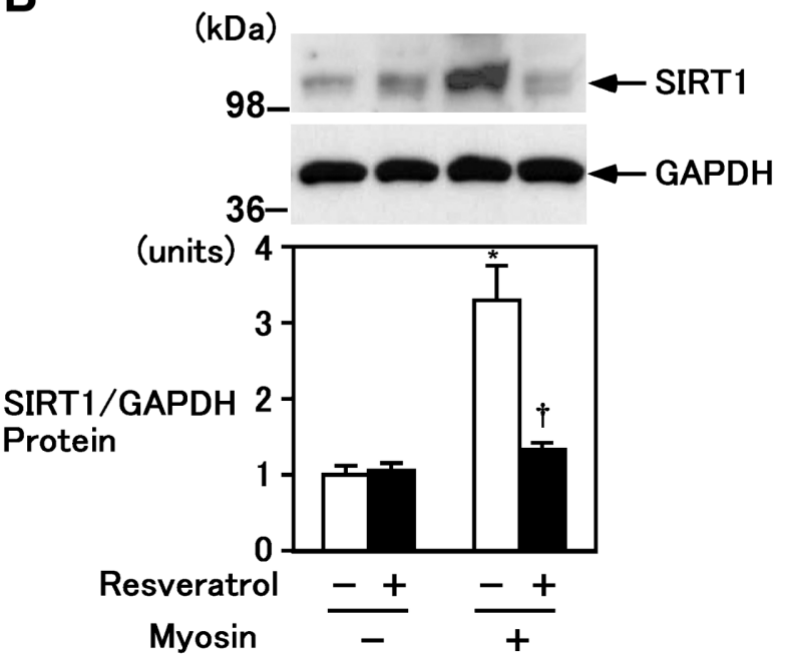

Fig 4. Expression of SIRT1, a mammalian ortholog of silent information regulator 2 protein in the heart of experimental model of autoimmune myocarditis. (A) SIRT1 mRNA expression measured by quantitative reverse transcription-polymerase chain reaction was increased in the hearts of myosin-immunized rats. (B) SIRT1 protein expression. Representative Western blot of SIRT1 (Upper panel), and the result of quantitative densitometric analysis (Lower panel). SIRT1 protein was increased in the hearts of myosin-immunized rats. Vehicletreated unimmunized rats $(n=4)$, resveratrol-treated unimmunized rats $(n=4)$, vehicle-treated myosin-immunized rats $(n=5)$, and resveratroltreated myosin-immunized rats $(n=5)$ were used for the analysis. ${ }^{*} \mathrm{p}<0.05$ vs unimmunized rats treated with vehicle, ${ }^{\dagger} \mathrm{p}<0.05$ vs vehicletreated myosin-immunized rats. The mean value of vehicle-treated unimmunized rat was defined as 1 unit. GAPDH, glyceraldehye-3phosphate dehydrogene.

\section{Effect of Resveratrol at 3 Weeks After Immunization}

Three weeks after immunization (ie, Protocol 2), the FS of resveratrol-treated rats was greater than that of vehicletreated rats $(66.7 \pm 3.0 \%$ vs $34.4 \pm 4.4 \%, \mathrm{p}<0.05$, Table 8$)$. The heart weight/tibial length ratio of the resveratroltreated rats was smaller compared with vehicle-treated rats $(25.4 \pm 3.5 \mathrm{mg} / \mathrm{mm}$ vs $36.0 \pm 2.3 \mathrm{mg} / \mathrm{mm}, \mathrm{p}<0.05$, Table 9$)$. Thus, resveratrol effectively prevented myocarditis at 3 weeks after the immunization.

\section{Effect of Resveratrol on EAM After Immunization}

When vehicle or resveratrol was started 1 day after immunization (ie, Protocol 3), the FS of resveratrol-treated rats was greater than that of the vehicle-treated rats $(63.8 \pm 3.0 \%$ vs $36.3 \pm 4.3 \%, \mathrm{p}<0.05$, Table 10 ). The heart weight/tibial length ratio of resveratrol-treated rats was smaller compared with vehicle-treated rats $(19.9 \pm 2.0 \mathrm{mg} / \mathrm{mm}$ vs $30.4 \pm$

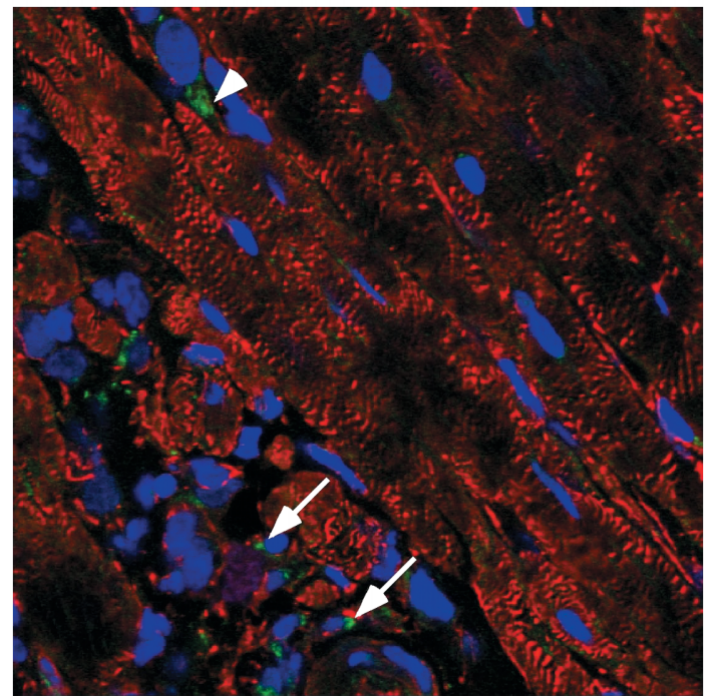

Fig 5. Localization of SIRT1 in the myocardium of experimental model of autoimmune myocarditis was examined by immunofluorescence analysis. The SIRT1 protein was mainly localized in infiltrating mononuclear cells (arrow). Some of the cardiac myocytes close to infiltrating mononuclear cells were also positive for SIRT1 immunoreactivity (arrowhead). Green, SIRT1; red, actinin; blue, nuclei.

Table 8 Echocardiographic Data of EAM Rats Treated With Resveratrol for 3 Weeks After Immunization

\begin{tabular}{lcc}
\hline \hline & Vehicle & Resveratrol \\
\hline No. animals & 10 & 8 \\
HR (beats/min) & $366 \pm 14$ & $400 \pm 7$ \\
Diastolic posterior wall thickness $(\mathrm{mm})$ & $2.63 \pm 0.21$ & $1.71 \pm 0.11^{*}$ \\
LV diastolic diameter $(\mathrm{mm})$ & $5.19 \pm 0.36$ & $4.68 \pm 0.14$ \\
LV systolic diameter $(\mathrm{mm})$ & $3.49 \pm 0.41$ & $1.58 \pm 0.19^{*}$ \\
FS $(\%)$ & $34.4 \pm 4.4$ & $66.7 \pm 3.0^{*}$ \\
\hline
\end{tabular}

Resveratrol was started 1 day before immunization, and given daily for 21 days after immunization.

Abbreviations see in Tables 1,2. Results are mean \pm SEM.

$* p<0.05$ vs vehicle-treated myosin-immunized rats.

Table 9 Postmortem Analysis of EAM Rats Treated With Resveratrol for 3 Weeks After Immunization

\begin{tabular}{lcc}
\hline \hline & Vehicle & Resveratrol \\
\hline No. animals & 10 & 10 \\
Body weight $(\mathrm{g})$ & $170.0 \pm 2.1$ & $181.9 \pm 5.2^{*}$ \\
Heart weight $(\mathrm{mg})$ & $1,224.3 \pm 77.5$ & $853.1 \pm 118.0^{*}$ \\
Lung weight $(\mathrm{mg})$ & $1,291.8 \pm 122.8$ & $1,194.6 \pm 110.7$ \\
Tibial length $(\mathrm{mm})$ & $34.1 \pm 0.2$ & $33.6 \pm 0.2^{*}$ \\
Heart weight/body weight $(\mathrm{mg} / \mathrm{g})$ & $7.24 \pm 0.51$ & $4.80 \pm 0.74^{*}$ \\
Lung weight/body weight $(\mathrm{mg} / \mathrm{g})$ & $7.59 \pm 0.71$ & $6.70 \pm 0.78$ \\
Heart weight/tibial length $(\mathrm{mg} / \mathrm{mm})$ & $36.0 \pm 2.3$ & $25.4 \pm 3.5^{*}$ \\
Lung weight/tibial length $(\mathrm{mg} / \mathrm{mm})$ & $38.0 \pm 3.7$ & $35.6 \pm 3.3$
\end{tabular}

Resveratrol was started 1 day before immunization, and given daily for 21 days after immunization.

Abbreviation see in Table 1. Results are mean \pm SEM.

$* p<0.05$ vs vehicle-treated myosin-immunized rats.

$2.4 \mathrm{mg} / \mathrm{mm}, \mathrm{p}<0.05$, Table 11). Thus, resveratrol also attenuated myocarditis when it was given after immunization.

\section{Discussion}

In this study, resveratrol preserved the cardiac function 
Table 10 Echocardiographic Data of EAM Rats Treated With Resveratrol Starting 1 Day After Immunization

\begin{tabular}{lcc}
\hline \hline & Vehicle & Resveratrol \\
\hline No. animals & 7 & 9 \\
HR $($ beats/min) & $380 \pm 17$ & $402 \pm 10$ \\
Diastolic posterior wall thickness $(\mathrm{mm})$ & $2.17 \pm 0.11$ & $1.58 \pm 0.49^{*}$ \\
LV diastolic diameter $(\mathrm{mm})$ & $4.89 \pm 0.24$ & $4.67 \pm 0.17$ \\
LV systolic diameter $(\mathrm{mm})$ & $3.10 \pm 0.21$ & $1.73 \pm 0.18^{*}$ \\
FS $(\%)$ & $36.3 \pm 4.3$ & $63.8 \pm 3.0^{*}$ \\
\hline
\end{tabular}

Resveratrol was started 1 day after immunization and given daily for 2 weeks.

Abbreviations see in Tables 1,2. Results are mean \pm SEM.

$* p<0.05$ vs vehicle-treated myosin-immunized rats.

of EAM rats. It attenuated the increase in heart weight and suppressed the expression of pro-inflammatory genes. The attenuation of myocardial injury was associated with a decrease in antioxidant gene expressions. SIRT1, a possible effector of resveratrol, was increased in the myocardium of EAM rats, and was mainly localized in infiltrating mononuclear cells.

In this study, we used DMSO as the solvent, because we needed to administer a relatively high dose of resveratrol, and it is known to have an immunomodulatory function? 24,25 We administered $0.5 \mathrm{ml}$ of DMSO or the same amount of saline to myosin-immunized rats for 14 days. The heart weight/tibial length ratio of DMSO-treated myosin-immunized rats was reduced compared with saline-treated myosin-immunized rats $(31.9 \pm 2.6 \mathrm{mg} / \mathrm{mm}$ vs $41.8 \pm 1.6 \mathrm{mg} / \mathrm{mm}$, $\mathrm{p}<0.05)$, although the effect of DMSO was much weaker than resveratrol dissolved in DMSO. Thus, it is possible that the strong suppression of the inflammatory response was a synergistic effect of DMSO and resveratrol.

The HR of myosin-immunized rats was higher than that of unimmunized rats on BP measurement without anesthesia, whereas that of myosin-immunized rats was lower than in unimmunized rats on echocardiographic analysis with anesthesia. It is likely that the response of myosin-immunized rats to anesthesia was different from that of unimmunized rats.

The mechanism of resveratrol's attenuation of EAM is unknown. Resveratrol is reported to reduce oxidative stress ${ }^{26,27}$ and in this study, the expression of oxidative stress-related genes $(\mathrm{Mn}-\mathrm{SOD}, \mathrm{Cu} / \mathrm{Zn}-\mathrm{SOD})$ were increased in the heart of myosin-immunized rats. This increase of both was likely the result of increased oxidative stress, and attenuation of myocarditis was associated with a decrease of Mn-SOD and $\mathrm{Cu} / \mathrm{Zn}-\mathrm{SOD}$. Thus, resveratrol may ameliorate EAM by reducing oxidative stress because oxygen radical scavengers are known to attenuate myocarditis.28,29

Alternatively, resveratrol may modulate EAM by acting on SIRT1, which was increased in the myocardium of myosin-immunized rats, and mainly localized in the infiltrating mononuclear cells. Fibroblasts deficient in SIRT1 proliferate more rapidly than wild-type cells 30 SIRT 1 induces cellcycle arrest by activating the Forkhead transcription factor $3^{1}$ Resveratrol also inhibits the proliferation of some tumors, 910 Thus, it is possible that resveratrol modulates the activity of SIRT1 in immune cells, and limits lymphocyte proliferation. However, the role of SIRT1 in immune cells is currently unknown and further studies are needed to clarify its role in EAM.

We used a relatively high dose of resveratrol. The $\mathrm{HW} / \mathrm{TL}$ ratio of myosin-immunized rats treated with a
Table 11 Postmortem Analysis of EAM Rats Treated With Resveratrol Starting 1 Day After Immunization

\begin{tabular}{lcc}
\hline \hline & Vehicle & Resveratrol \\
\hline No. animals & 9 & 9 \\
Body weight $(\mathrm{g})$ & $166.8 \pm 1.9$ & $167.1 \pm 2.8$ \\
Heart weight $(\mathrm{mg})$ & $1,012.7 \pm 77.2$ & $668.3 \pm 68.2^{*}$ \\
Lung weight $(\mathrm{mg})$ & $1,117.3 \pm 29.6$ & $988.0 \pm 31.2^{*}$ \\
Tibial length $(\mathrm{mm})$ & $33.4 \pm 0.2$ & $33.6 \pm 0.2$ \\
Heart weight/body weight $(\mathrm{mg} / \mathrm{g})$ & $6.06 \pm 0.45$ & $4.02 \pm 0.43^{*}$ \\
Lung weight/body weight $(\mathrm{mg} / \mathrm{g})$ & $6.71 \pm 0.23$ & $5.90 \pm 0.14^{*}$ \\
Heart weight/tibial length $(\mathrm{mg} / \mathrm{mm})$ & $30.4 \pm 2.4$ & $19.9 \pm 2.0^{*}$ \\
Lung weight/tibial length $(\mathrm{mg} / \mathrm{mm})$ & $33.5 \pm 0.9$ & $29.4 \pm 0.9^{*}$ \\
\hline
\end{tabular}

Resveratrol was started 1 day after immunization, and given daily for 2 weeks.

Abbreviation see in Table 1. Results are mean \pm SEM.

$*_{p}<0.05$ vs vehicle-treated myosin-immunized rats.

lower dose of resveratrol $(10 \mathrm{mg} / \mathrm{kg}$ per day) was not different from that of vehicle-treated myosin-immunized rats $(43.9 \pm 6.7 \mathrm{mg} / \mathrm{mm}$ vs $43.3 \pm 3.9 \mathrm{mg} / \mathrm{mm})$. Resveratrol is reported to have different effects on immune cells, depending on the dose and experimental condition? 32,33

In conclusion, resveratrol effectively ameliorated EAM. Although further study is needed, resveratrol may be a therapeutic modality for myocarditis.

\section{Acknowledgments}

This study was supported in part by grants from the Japan Society for the Promotion of Science (15390252), the Japan Heart Foundation, the Japan Foundation of Cardiovascular Research, the NOVARTIS Foundation for the Promotion of Science, the Mochida Memorial Foundation for Medical and Pharmaceutical Research, the Takeda Medical Research Foundation, the Kanagawa Nanbyo Foundation, the Takeda Science Foundation (to T.S.), and the Japan Society for the Promotion of Science (16590713), Postgraduate Research Project of Kitasato University (to T.I.).

\section{References}

1. Drory Y, Turetz Y, Hiss Y, Lev B, Fisman EZ, Pines A, et al. Sudden unexpected death in persons less than 40 years of age. Am J Cardiol 1991; 68: $1388-1392$.

2. Hiramitsu S, Morimoto S, Kato S, Uemura A, Ohtsuki M, Kato Y, et al. Clinical course of myocarditis through the acute, fulminant and fatal chronic stages: An autopsy case. Circ J 2006; 70: 1086-1090.

3. Woodruff JF. Viral myocarditis: A review. Am J Pathol 1980; 101: 425-484.

4. Lauer B, Padberg K, Schultheiss HP, Strauer BE. Autoantibodies against human ventricular myosin in sera of patients with acute and chronic myocarditis. J Am Coll Cardiol 1994; 23: 146-153.

5. Kodama M, Matsumoto Y, Fujiwara M, Masani F, Izumi T, Shibata A. A novel experimental model of giant cell myocarditis induced in rats by immunization with cardiac myosin fraction. Clin Immunol Immunopathol 1990; 57: 250-262.

6. Kawada H, Niwano S, Niwano H, Yumoto Y, Wakisaka Y, Yuge M, et al. Tumor necrosis factor $\boldsymbol{\alpha} \cdot$ downregulates the voltage gated outward $\mathrm{K}+$ current in cultured neonatal rat cardiomyocytes: A possible cause of electrical remodeling in diseased hearts. Circ J 2006; 70: 605-609.

7. Ishiyama S, Hiroe M, Nishikawa T, Abe S, Shimojo T, Ito H, et al. Nitric oxide contributes to the progression of myocardial damage in experimental autoimmune myocarditis in rats. Circulation 1997; 95: 489-496.

8. Sato M, Maulik N, Das DK. Cardioprotection with alcohol: Role of both alcohol and polyphenolic antioxidants. Ann NY Acad Sci 2002; 957: $122-135$.

9. Shazib P. Resveratrol: From grapevines to mammalian biology. FASEB J 2003; 17: 1975-1985.

10. Lucie F. Biological effects of resveratrol. Life Sci 2000; 66: 663673.

11. Dernek S, Ikizler M, Erkasap N, Ergun B, Koken T, Yilmaz K, et al. Cardioprotection with resveratrol pretreatment: Improved beneficial 
effects over standard treatment in rat hearts after global ischemia. Scand Cardiovasc J 2004; 38: 245-254.

12. Ray PS, Maulik G, Cordis GA, Bertelli AA, Bertelli A, Das DK. The red wine antioxidant resveratrol protects isolated rat hearts from ischemia reperfusion injury. Free Radic Biol Med 1999; 27: 160 169.

13. Cheng TH, Liu JC, Lin H, Shih NL, Chen YL, Huang MT, et al. Inhibitory effect of resveratrol on angiotensin II-induced cardiomyocyte hypertrophy. Naunyn Schmiedebergs Arch Pharmcol 2004; 369: 239-244.

14. Shigeaki K, Lijun Z, Masahiko M, Nilanjana M. Resveratrol enhances neovascularization in the infarcted rat myocardium through the induction of thioredoxin-1, heme oxigenase-1 and vascular endothelial growth factor. J Mol Cell Cardiol 2005; 39: 813-822.

15. Blander G, Guarente L. The Sir2 family of protein deacetylases. Annu Rev Biochem 2004; 73: 417-435.

16. Hiroaki D, Mitsutoki H, Hitomi M, Satoko A, Takayuki O, Makoto M, et al. Silent information regulator 2 potentiates Foxo1-mediated transcription through its deacetylase actyvity. Proc Natl Acad Sci USA 2004; 101: $10042-10047$

17. Leonard G, Frederic P. Calorie Restriction-the Sir2 connection. Cell 2005; 120: $473-482$.

18. Araki T, Sasaki Y, Milbrandt J. Increased nuclear NAD biosynthesis and SIRT1 activation prevent axonal degeneration. Science 2004; 305: $1010-1013$.

19. Howitz KT, Bitterman KJ, Cohen HY, Lamming DW, Lavu S, Wood JG, et al. Small molecule activators of sirtuins extend Saccharomyces cerevisiae lifespan. Nature 2003; 425: 191-196.

20. Inomata $\mathrm{T}$, Hanawa $\mathrm{H}$, Miyanishi $\mathrm{T}$, Yajima E, Nakayama S, Maita $\mathrm{T}$, et al. Localization of porcine cardiac myosin epitopes that induce experimental autoimmune myocarditis. Circ Res 1995; 76: 726 733 .

21. Bunag RD. Validation in awake rats of a tail-cuff method for measuring systolic pressure. J Appl Physiol 1973; 34: 279-282.

22. Shioi T, Kang PM, Douglas PS, Hampe J, Yballe CM, Lawitts J, et al. The conserved phosphoinositide 3-kinase pathway determines heart size in mice. EMBO J 2000; 19: 2537-2548.

23. Kameda Y. Mash1 is required for glomus cell formation in the mouse carotid body. Dev Biol 2005; 283: 128-139.

24. Milner L, de Chadarevian J, Goodyer P, Mills M, Kaplan B. Amelioration of murine lupus nephritis by dimethylsulfoxide. Clin Immunol Immunopathol 1987; 45: 259-267.

25. Pestronk A, Teoh R, Sims C, Drachman DB. Effects of dimethylsulfoxide on humoral immune responses to acetylcholine receptors in the rat. Clin Immunol Immunopathol 1985; 37: 172-178.

26. Shigematsu S, Ishida S, Hara M, Takahashi N, Yoshimatsu H, Sakata $\mathrm{T}$, et al. Resveratrol, a red wine constituent polyphenol, prevents superoxide-dependent inflammatory responses induced by ischemia/reperfusion, platelet-activating factor, or oxidants. Free Radic Biol Med 2003; 34: 810-817.

27. Sengottuvelan M, Viswanathan P, Nalini N. Chemopreventive effect of trans-resveratrol: A phytoalexin against colonic aberrant crypt foci and cell proliferation in 1,2-dimethylhydrazine induced colon carcinogenesis. Carcinogenesis 2006; 27: 1038-1046.

28. Suzuki H, Matsumori A, Matoba Y, Kyu BS, Tanaka A, Fujita J, et al. Enhanced expression of superoxide dismutase messenger RNA in viral myocarditis: An SH-dependent reduction of its expression and myocardial injury. J Clin Invest 1993; 91: 2727-2733.

29. Hiraoka Y, Kishimoto C, Kurokawa M, Ochiai H, Sasayama S. Effects of polyethylene glycol conjugated superoxide dismutase on coxsackievirus B3 myocarditis in mice. Cardiovasc Res 1992; 26: 956-961.

30. Chua KF, Mostoslavsky R, Lombard DB, Pang WW, Saito S, Franco $\mathrm{S}$, et al. Mammalian SIRT1 limits replicative life span in response to chronic genotoxic stress. Cell Metab 2005; 2: 67-76.

31. Brunet A, Sweeney LB, Sturgill JF. Chua KF, Greer PL, Lin Y, et al. Stress-dependent regulation of FOXO transcription factors by the SIRT1 deacetylase. Science 2004; 303: 2011-2015.

32. Gao X, Deeb D, Media J, Divine G, Jiang H, Chapman RA, et al. Immunomodulatory activity of resveratrol: Discrepant in vitro and in vivo immunological effects. Biochem Pharmacol 2003; 66: 2427 2435.

33. Feng Y, Zhou W, Wu Q, Li X, Zhao W, Zou J. Low dose of resveratrol enhanced immune response of mice. Acta Pharmacol Sin 2002; 23: $893-897$. 